\title{
Refinement of response assessment in neuro-oncology (RANO) using non- enhancing lesion type and contrast enhancement evolution pattern in IDH wild-type glioblastomas
}

\author{
Hye Hyeon Moon ${ }^{1}$, Ho Sung Kim, Ji Eun Park', Young-Hoon Kim² and Jeong Hoon Kim²
}

\begin{abstract}
Background: Updated response assessment in neuro-oncology (RANO) does not consider peritumoral nonenhancing lesion (NEL) and baseline (residual) contrast enhancement (CE) volume. The objective of this study is to explore helpful imaging characteristics to refine RANO for assessing early treatment response (pseudoprogression and time-to-progression [TP]) in patients with IDH wild-type glioblastoma.

Methods: This retrospective study enrolled 86 patients with IDH wild-type glioblastoma who underwent consecutive MRI examinations before and after concurrent chemoradiotherapy (CCRT). NEL was classified as edema- or tumordominant type on pre-CCRT MRI. CE evolution was categorized into 4 patterns based on post-operative residual CE (measurable vs. non-measurable) and CE volume change (same criteria with RANO) during CCRT. Multivariable logistic regression, including clinical parameters, NEL type, and CE evolution pattern, was used to analyze pseudoprogression rate. TTP and OS according to NEL type and CE evolution pattern was analyzed by the Kaplan-Meier method.

Results: Pseudoprogression rate was significantly lower (chi-square test, $P=.047$ ) and TTP was significantly shorter (hazard ratio $[H R]=2.03, P=.005$ ) for tumor-dominant type than edema-dominant type of NEL. NEL type was the only predictive marker of pseudoprogression on multivariate analysis (odds ratio $=0.26, P=.046$ ). Among CE evolution patterns, TTP and OS was shortest in patients with residual CE compared with those exhibiting new CE $(H R=4.33$, $P<0.001$ and $H R=3.71, P=.009$, respectively). In edema-dominant NEL type, both TTP and OS was stratified by CE evolution pattern (log-rank, $P=.001$ ), whereas it was not in tumor-dominant NEL.

Conclusions: NEL type improves prediction of pseudoprogression and, together with CE evolution pattern, further stratifies TTP and OS in patients with IDH wild-type glioblastoma and may become a helpful biomarker for refining RANO.
\end{abstract}

Keywords: Progression, Glioblastoma, MRI, Non-enhancing lesion, Contrast enhancement

\footnotetext{
* Correspondence: radhskim@gmail.com

'Department of Radiology and Research Institute of Radiology, University of

Ulsan College of Medicine, Asan Medical Center, 43 Olympic-ro 88, 86

Asanbyeongwon-Gil, Songpa-Gu, Seoul 05505, Republic of Korea

Full list of author information is available at the end of the article
} 


\section{Background}

The Response Assessment in Neuro-oncology (RANO) criteria are accurate and reproducible in the radiologic assessment of treatment response in patients with glioblastoma [1]. RANO criteria focus primarily on objective measurements of contrast enhancement $(\mathrm{CE})$, whereas the importance of non-contrast enhancing components of tumors is frequently overlooked [2-4]. Peritumoral non-enhancing lesions (NELs), which appear as hyperintense lesions on T2-weighted fluid-attenuated inversion recovery (FLAIR) imaging [5], usually consist of mixtures of edema and tumor [6]. Because glioblastomas are infiltrative, NELs can affect patient prognosis [7-9], as they represent the portion of tumors extending beyond the contrast-enhanced margins.

Although advanced magnetic resonance imaging (MRI) protocols, including perfusion MRI, diffusionweighted imaging (DWI), and MR spectroscopy, have shown potential for distinguishing between tumor and edema in NELs $[10,11]$, these methods require additional postprocessing method and are not easily applied in clinical practice $[12,13]$. Attempts have been made to distinguish infiltrative tumor from edema in NEL by their morphology on anatomical MRI [14]. Tumordominant NELs have several characteristic imaging features, including relatively mild FLAIR hyperintensity, gray matter involvement, eccentric extension not accounted for by anatomic constraints, focal parenchymal expansion, and mass effect [14-16].

The post-operative residual $\mathrm{CE}$ volume is another important prognostic marker, as extent of surgical resection has been associated with overall survival [17, 18]. However, RANO criteria did not substratify evolution of $\mathrm{CE}$ after treatment, whether responses are determined by comparisons with residual measurable $\mathrm{CE}$ or nonmeasurable CE. For example, stable disease (SD) can indicate an absence of change in residual measurable $\mathrm{CE}$ on follow-up examination, or no $\mathrm{CE}$ at all, without change on follow-up. Assessment of response to CCRT may be refined by evaluating CE and NEL before and after treatment. The present study evaluated whether categorizing NEL type and CE evolution pattern during CCRT can better predict time to progression (TTP) and pseudoprogression in patients with isocitrate dehydrogenase (IDH) wild-type glioblastoma.

\section{Materials and methods}

\section{Study population}

This retrospective clinical study was approved by the institutional review board (IRB) of Asan Medical Center (local approval number: 2019-0135) and was conducted in compliance with the U.S. Health Insurance Portability and Accountability Act (HIPAA) regulations and the Declaration of Helsinki. The requirement of written informed consent was waived in this retrospective study as per IRB-approval protocol. The Asan Medical Center database was retrospectively reviewed to identify consecutive patients who were confirmed as having glioblastoma between July 2011 and August 2019, and were evaluated by MRI after surgery followed by CCRT. Patients were included if they (i) had been histologically diagnosed with IDH wild-type glioblastoma according to the World Health Organization (WHO) criteria; (ii) underwent adjuvant CCRT that included six cycles of temozolomide (TMZ) treatment after surgical resection or biopsy; (iii) had been evaluated by MRI, including contrast-enhanced T1-weighted imaging (CE-T1WI) and FLAIR imaging, within 2 weeks after surgery or biopsy and before CCRT, and again 4 weeks after completing CCRT; (iv) had a newly developed or persistent CE on post-CCRT MRI; and (vi) were sequentially followed-up by contrast-enhanced MRI at 2-3 month intervals for at least 12 months to confirm the final diagnosis of pseudoprogression and progression. The protocol of this retrospective study was approved by the institutional review board of Asan Medical Center, which waived the requirement for patient informed consent (approval number: 2019-1259). A study flowchart is shown in Fig. 1.

\section{Molecular/genomic analysis IDH mutation status analysis}

Immunohistochemistry for IDH1 (R132H) protein expression was used as the reference standard for this study. In patients older than 55 years with typical newly identified glioblastoma at diagnosis, a negative immunohistochemistry result for IDH1 (R132H) expression was sufficient for IDH wild-type glioblastoma classification, according to the European Association of NeuroOncology guidelines. In patients younger than 55 years at diagnosis, DNA pyrosequencing for mutations in IDH1 or IDH2 genes was performed [19].

\section{MGMT promoter methylation analysis}

MGMT promoter methylation status was evaluated using a methylation-specific polymerase chain reaction (PCR) assay [20]. The genomic DNA was extracted from an unstained tissue slide made from paraffin-embedded blocks of tumor tissue. DNA methylation status of $\mathrm{CpG}$ (cytosine and guanine nucleotides separated by one phosphate nucleotide) islands at the MGMT promoter was evaluated using the methylation-specific PCR assay with some modifications [21]. Bisulfite-treated unmethylated DNA and methylated DNA (Qiagen, Hilden, Germany) were used as low and high controls. PCR products were separated on $8 \%$ polyacrylamide gels and stained with ethidium bromide. Subsequently, they were examined under ultraviolet illumination. The clinical 


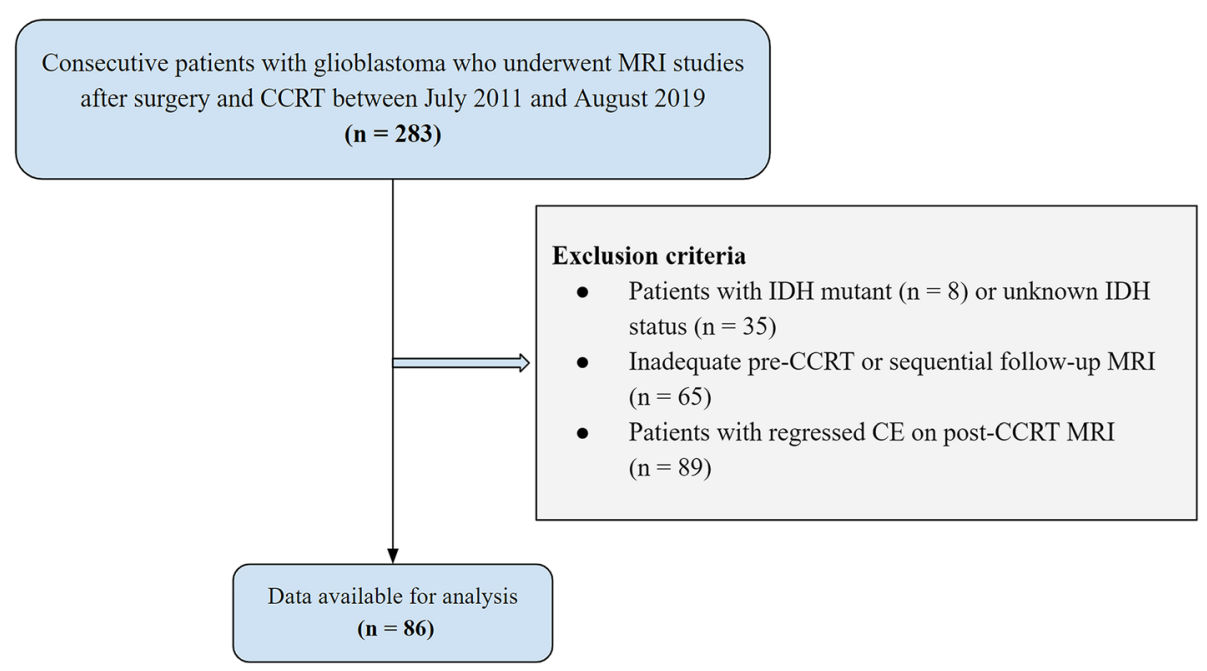

Fig. 1 Study flow chart showing included and excluded patients. CCRT = concurrent chemoradiotherapy; IDH = isocitrate dehydrogenase

information was blinded with a computerized barcode during all these processes.

\section{Reference standard for final diagnosis}

Pseudoprogression and true progression were pathologically confirmed on second-look operations when clinically indicated. In the absence of second-look operations, consecutive clinicoradiological diagnoses were made by consensus between two neuro-oncologists (J.H.K., Y.H.K; with 28 and 10 years of clinical experience in neuro-oncology, respectively) according to RANO criteria [4]. Pseudoprogression was defined as an increase in CE or any new lesion followed by stabilization or regression without any changes in treatment for at least 6 months after surgery and completion of CCRT [4]. True progression was defined as the occurrence of any new lesion outside the radiation field or a gradual increase in CE size on more than two subsequent follow-up MRI examinations performed at 2-3 month intervals and requiring a prompt change in treatment [4]. TTP was defined as the time from the date of initial diagnosis to the date of first documented progression [22]. The co-primary endpoint was overall survival (OS). OS was calculated from the day of histopathologic diagnosis until the day of death as obtained from the national health care data linked to our hospital. Patients who were alive at the time of analysis $(n=17,19.7 \%)$ with right-censored data were included in the analysis. All patients were followed up every 3-6 months.

\section{Magnetic resonance imaging}

All MRI evaluations were performed using a 3-T unit (Ingenia $3.0 \mathrm{CX}$; Philips Healthcare, Best, the Netherlands) with a 16-channel head coil, and included the following sequences: T2-weighted, T2-weighted
FLAIR, and precontrast and postcontrast T1-weighted images. T2-weighted and FLAIR images were acquired using a spin echo sequence with the following parameters: repetition time (TR)/echo time (TE) 3000/100 ms, FOV $240 \times 240 \mathrm{~mm}$; matrix, $256 \times 256$; slice thickness, 4 $\mathrm{mm}$ without a gap for T2-weighted image and TR/TE $10000 / 130 \mathrm{~ms}$, inversion time $2800 \mathrm{~ms}$, FOV $240 \times 240$ $\mathrm{mm}$; matrix, $256 \times 256$; and slice thickness, $4 \mathrm{~mm}$ without a gap for FLAIR. High-resolution anatomic threedimensional (3D) volume images were acquired using gradient-echo $\mathrm{T} 1$-weighted sequences with the following parameters: TR/TE $9.8 / 4.6 \mathrm{~ms}$; flip angle, $10^{\circ}$; FOV, $256 \times 256 \mathrm{~mm}$; matrix, $512 \times 512$; and slice thickness, 1 $\mathrm{mm}$ with no gap, with and without gadolinium-based contrast agent.

\section{Image analysis}

All images were analyzed on T2 FLAIR imaging and CET1WI by two neuroradiologists (H.S.K. and J.E.P.; with 22 and 7 years of clinical experience, respectively, in neuro-oncologic imaging), who were blinded to the clinical information and reference standard.

\section{Type of NEL on pre-CCRT examinations}

NELs, visualized as T2 FLAIR hyperintense lesions on pre-CCRT MRI, were classified as edema-dominant or tumor-dominant type. Characteristic MRI features for tumor-dominant type NEL were relatively mild FLAIR hyperintensity, gray matter involvement, eccentric extension not accounted for by anatomic constraints, focal parenchymal expansion, and mass effect [14-16] (Fig. 2A). The two neuroradiologists evaluated MRI results independently, and agreement was calculated. For multivariate analysis, disagreements were reconciled by consensus. 

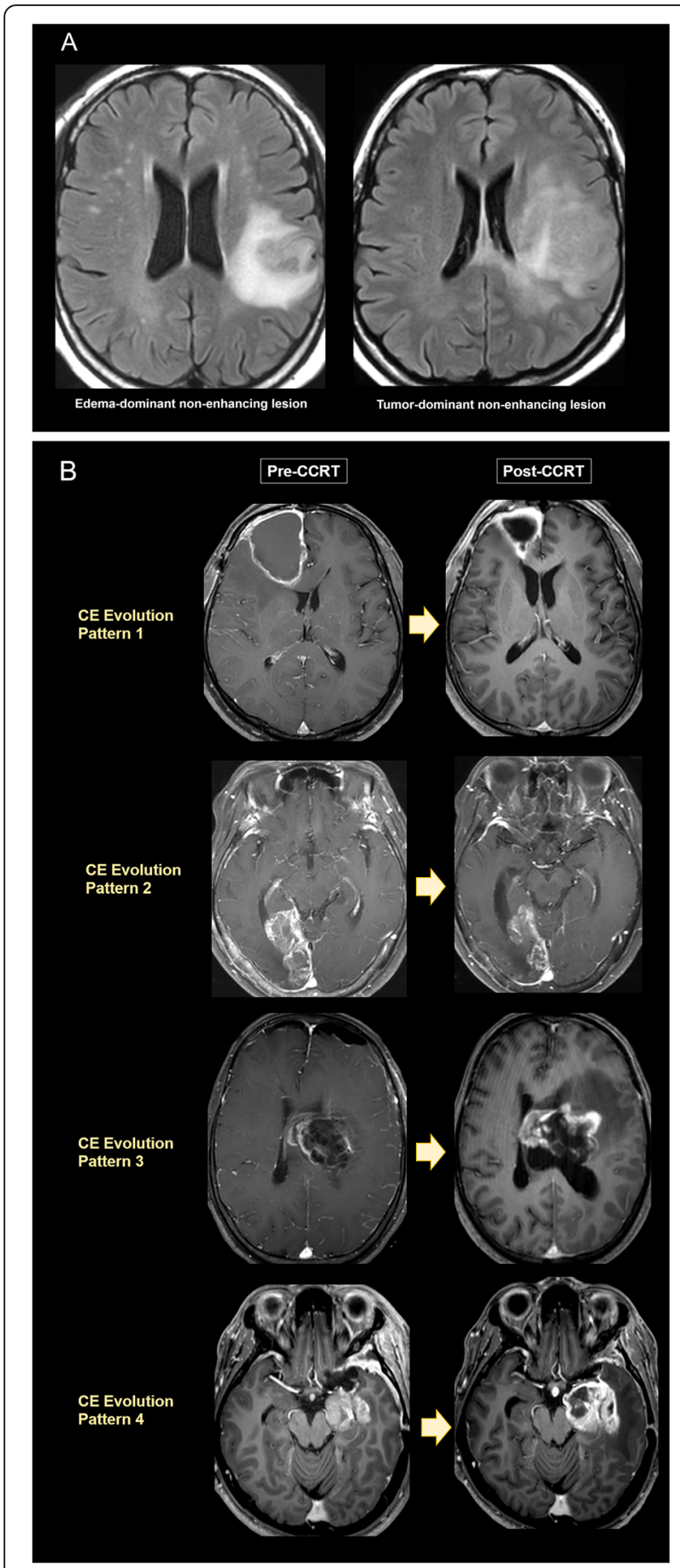

Fig. 2 (A) Representative patient images for classification of NEL type based on pre-CCRT T2 FLAIR images. Edema-dominant type NEL (left) and tumor-dominant type NEL (right). (B) Representative patient images for CE evolution pattern based on pre-CCRT (left) and post-CCRT (right) contrastenhanced T1-weighted MRI. a) new non-measurable CE after GTR (pattern 1); b) stable or responding residual CE after STR (pattern 2); c) new measurable CE after GTR (pattern 3); and d) enlarging residual CE after STR (pattern 4). NEL = non-enhancing lesion; $C E=$ contrast enhancing lesion; $\mathrm{CCRT}=$ concurrent chemoradiotherapy; GTR = gross total resection; $\mathrm{STR}=$ subtotal resection

\section{Evolution pattern of CE from pre- to post-CCRT examinations}

CEs, visualized on CE-T1WI, were categorized by two readers in consensus as (i) new non-measurable $\mathrm{CE}$ after gross total resection (GTR) (pattern 1); (ii) stable or responding residual $\mathrm{CE}$ after subtotal resection (STR) (pattern 2); (iii) new measurable CE after GTR (pattern 3); and (iv) and enlarging residual CE after STR (pattern 4) (Fig. 2B). Measurable CE was defined as bidimensional CE with clearly defined margins, with two perpendicular diameters of at least $10 \mathrm{~mm}$, visible on two or more axial slices [4]. Non-measurable CE was defined as the absence of clearly defined margins, unidimensional measurable lesions, or lesion maximal perpendicular diameters $<10 \mathrm{~mm}$ [4]. Enlarging CE was defined as a $>$ $25 \%$ increase from baseline in the sum of the products of perpendicular diameters of $\mathrm{CE}$.

\section{Statistical analysis}

The demographic and clinical characteristics of the edema-dominant and tumor-dominant NEL groups were assessed by Student's t-test or the chi-square test, as appropriate.

\section{Inter-reader agreement}

The inter-reader agreement on NEL type was assessed by using kappa statistics.

\section{Prediction of TTP}

The effects of NEL type and CE evolution pattern on TTP and OS were evaluated by the Kaplan-Meier method and compared by log-rank tests and by Cox proportional hazard regression models. The effects of $\mathrm{CE}$ evolution pattern on TTP and OS in the two NEL subgroups were also analyzed by the Kaplan-Meier method and compared by log-rank tests.

\section{Prediction of pseudoprogression}

Differences in pseudoprogression rates according to NEL type and CE evolution pattern were assessed by chi-square tests and multivariable logistic regression analysis, after adjusting for age, sex, and Karnofsky performance status (KPS) at baseline. The diagnostic accuracy of predicting pseudoprogression was analyzed according to NEL type and CE evolution pattern, both independently and as a combination of NEL type and CE evolution pattern.

All statistical analyses were performed using MedCalc version 19.2.1 and $\mathrm{R}$ version 3.6.3 statistical software, with $P$-values $<.05$ considered statistically significant.

\section{Results}

Patient demographics

Of 283 potentially eligible patients, 43 patients with IDH-mutant type or unknown IDH status, 65 patients 
without appropriate pre-CCRT or sequential follow-up MRI, and 89 patients with regressed CE on post-CCRT MRI were excluded. Finally, 86 patients, 49 (57.0\%) men and 37 (43.0\%) women, of mean age 58 years, were included in the analysis. Of these 86 patients, 41 (47.7\%) were classified as edema-dominant type NEL group and $45(52.3 \%)$ as tumor-dominant type NEL group. Final diagnosis for pseudoprogression and true progression was based on pathologic confirmation for 16 (18.6\%) patients and clinicoradiologic follow-up for 70 (81.4\%) patients. There were no significant differences between groups of patients with edema-dominant type and tumor-dominant type NEL in age at diagnosis, sex, KPS at baseline, and O6-methylguanine-DNA methyltransferase (MGMT) promoter methylation status (Table 1). On the other hand, edema-dominant NEL had a higher rate of GTR $(P=.02)$ than tumor-dominant NEL group.

\section{Prediction of Pseudoprogression}

Of the 86 patients, 34 (39.5\%) showed pseudoprogression. In patients with CE evolution pattern 3 and 4, pseudoprogression rate was lower in the tumor-dominant type than in the edema-dominant type NEL group (chi-square $=3.940, P=$ .047). Multivariate analyses that included age, sex, KPS, NEL type, and CE evolution pattern showed that NEL type was the only independent predictive marker for pseudoprogression (odds ratio $[\mathrm{OR}]=0.26,95 \% \mathrm{CI}=0.00-0.52, P=.046$ ). $\mathrm{CE}$ evolution pattern was not associated with pseudoprogression $(P=.407)$. Accuracy of a combination of NEL type and $\mathrm{CE}$ evolution patterns in predicting pseudoprogression was shown in the Supplementary Table 1. The edema-dominant type NEL could predict pseudoprogression with an accuracy of $62.8 \%$, while the tumor-dominant type NEL could do the same with an accuracy of only $38.4 \%$. After combining NEL type and CE evolution pattern, the accuracy of predicting pseudoprogression was highest in edema-dominant type NEL with CE evolution pattern 3 (62.8\%), followed by edema-dominant type NEL with CE evolution pattern 1 (61.6\%), tumor-dominant type NEL with CE evolution pattern 1 (60.5\%), edema-dominant type NEL with CE evolution pattern 2 (59.3\%), edema-dominant type NEL with CE evolution pattern 4 (59.3\%), tumor-dominant type NEL with CE evolution pattern 2 (59.3\%), tumor-dominant type NEL with CE evolution pattern 4 (53.5\%), and tumor-dominant type NEL with CE evolution pattern 3 (46.5\%).

\section{Independent prediction of TTP and OS based on NEL type and CE evolution pattern}

The kappa value for interobserver agreement on type of NEL was 0.81 (95\% confidence interval $[\mathrm{CI}]=0.69-0.94)$, indicating almost perfect agreement.

Table 2 summarizes the association between imaging predictors and TTP. Evaluation of all study patients showed that TTP was significantly shorter in patients with tumor-dominant type than edema-dominant type NEL (hazard ratio $[\mathrm{HR}]=2.03, P=.005$ ). OS tended to be shorter in patients with tumor-dominant type than with edema-dominant type NEL. However, this difference did not reach statistical significance $(\mathrm{HR}=1.37$, $P=$.196) (Supplementary Fig. 1).

Stratification by CE evolution pattern showed that TTP was shortest in patients with enlarging residual $\mathrm{CE}$ after STR (pattern 4; $\mathrm{HR}=4.33, P<.001$ ), followed by new measurable CE after GTR (pattern 3; HR = 3.00, $P<.001$ ), stable or responding residual CE after STR (pattern 2; $\mathrm{HR}=2.44, P=.117)$, and new non-measurable CE after GTR (pattern $1 ; P<.001$ ). Stratification by CE evolution pattern revealed that the shortest OS was found in patients with pattern $2(\mathrm{HR}=3.71, P=.009)$, followed by pattern $4(\mathrm{HR}=2.74, P=.04)$, pattern $3(\mathrm{HR}=2.50, P=$ $.005)$, and pattern $1(P<.001)$ (Supplementary Fig. 2).

\section{Prediction of TTP and OS based on the relationship between NEL type and CE evolution pattern Relationship between NEL type and CE evolution pattern}

Of the 41 patients with edema-dominant type NEL, 19 were classified as new non-measurable CE after GTR (pattern 1), one as stable or responding residual CE after

Table 1 Demographic Characteristics of the Study Population

\begin{tabular}{|c|c|c|c|}
\hline & $\begin{array}{l}\text { Edema-dominant } \\
\text { NEL Group }(n=41)\end{array}$ & $\begin{array}{l}\text { Tumor-dominant } \\
\text { NEL Group }(n=45)\end{array}$ & $P$ \\
\hline Age, years, median (range) & $59(31-81)$ & $57(31-77)$ & .362 \\
\hline No. of women patients (\% of patients) & $15(36.6 \%)$ & $22(48.9 \%)$ & .253 \\
\hline KPS at baseline $\geq 70$ (\% of patients) & $38(92.7 \%)$ & $40(88.9 \%)$ & .548 \\
\hline MGMT promoter methylation (positive/negative/missing) & $18 / 19 / 4$ & 16/19/10 & .285 \\
\hline Type of surgery (biopsy/STR/GTR) & $0 / 4 / 37$ & $3 / 12 / 30$ & .023 \\
\hline No. with pseudoprogression (\% of patients) & $21(51.2 \%)$ & $13(28.9 \%)$ & .036 \\
\hline No. with true progression (\% of patients) & $20(48.8 \%)$ & $32(71.1 \%)$ & .119 \\
\hline Median TTP, days & 257 (95\% Cl: 205.9-305.5) & 105 (95\% Cl: 98.5-153.6) & .042 \\
\hline
\end{tabular}

NEL non-enhancing lesion, IDH isocitrate dehydrogenase, KPS Karnofsky performance status, MGMT O6-methylguanine-DNA methyltransferase, STR subtotal resection, GTR gross total resection, $\mathrm{Cl}$ confidence interval, TTP time to progression 
Table 2 Univariate Cox Proportional Hazard Analysis of Prediction of TTP in Patients Stratified by NEL Type and CE Evolution Pattern

\begin{tabular}{|c|c|c|c|}
\hline Entire Patients $(n=86)$ & Hazard Ratio & $95 \% \mathrm{Cl}$ & $P$ \\
\hline \multicolumn{4}{|l|}{ NEL type } \\
\hline Tumor-dominant & 2.03 & $1.24-3.32$ & .005 \\
\hline Edema-dominant & 1 & & \\
\hline \multicolumn{4}{|l|}{ CE evolution pattern } \\
\hline Enlarging residual CE (pattern 4) & 4.33 & $2.02-9.28$ & $<.001$ \\
\hline New measurable CE (pattern 3) & 3.00 & $1.63-5.54$ & $<.001$ \\
\hline Stable or responding residual CE (pattern 2) & 2.44 & $0.80-7.28$ & .117 \\
\hline New non-measurable CE (pattern 1) & 1 & & \\
\hline \multicolumn{4}{|l|}{ Patients with edema-dominant NEL $(n=41)$} \\
\hline Enlarging residual CE (pattern 4) & 6.92 & $1.71-27.93$ & .007 \\
\hline New measurable CE (pattern 3) & 5.36 & $2.10-13.66$ & $<.001$ \\
\hline Stable or responding residual CE (pattern 2) & 2.63 & $0.31-21.98$ & .372 \\
\hline New non-measurable CE (pattern 1) & 1 & & \\
\hline
\end{tabular}

TTP time to progression, NEL non-enhancing lesion, $C E$ contrast enhancement, $C l$ confidence interval

STR (pattern 2), 18 as new measurable CE after GTR (pattern 3), and three as enlarging residual CE after STR (pattern 4). Of the 45 patients with tumor-dominant type NEL, four, three, 26, and 12 were classified as having $1,2,3$, and $4 \mathrm{CE}$ evolution pattern, respectively. NEL type was significantly associated with $\mathrm{CE}$ evolution pattern (chi-square $=11.963, P=.008$, Fig. 3 ). A high proportion of patients with edema-dominant type NEL had CE evolution pattern 1 and 3, whereas a proportion of patients with tumor-dominant type NEL had CE evolution pattern 3 and 4 .

\section{Prediction of TTP and OS based on NEL type and CE evolution pattern}

Subgroup analysis showed that TTP differed significantly in patients with edema-dominant type NEL according to CE evolution pattern. TTP was shortest in patients with CE evolution pattern $4(\mathrm{HR}=6.92, P=.007)$, followed by pattern $3(\mathrm{HR}=5.36, P<.001)$, pattern $2(\mathrm{HR}=2.63, P=$ .372 ), and pattern 1 (log-rank, $P=.001$ ). TTP in patients with tumor-dominant type NEL, however, did not differ significantly according to CE evolution pattern (log-rank, $P=.528)$.

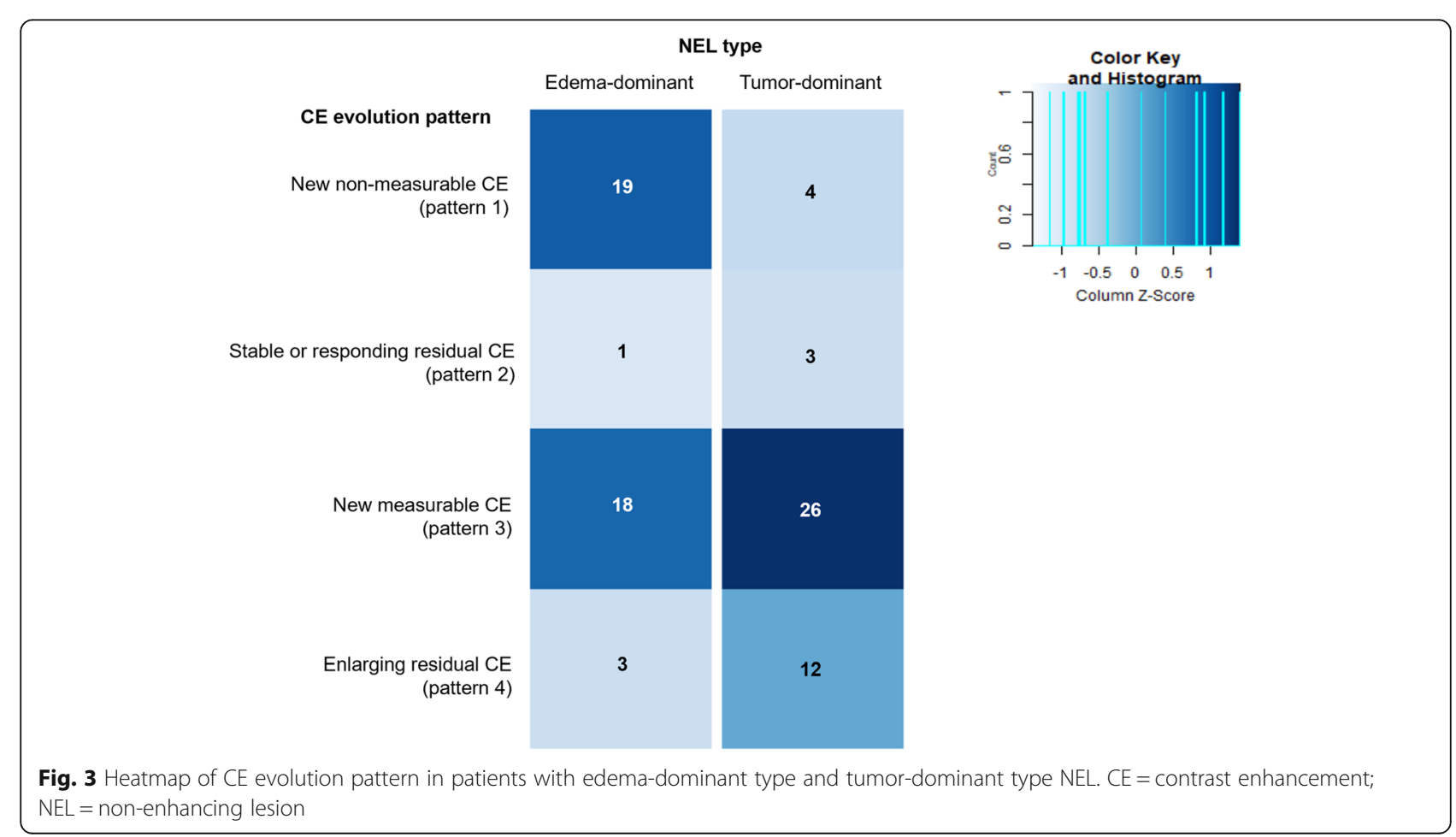


With OS, the same trend was observed. In patients with edema-dominant type NEL, OS was stratified by CE evolution pattern, shortest in patients with pattern 2 $(\mathrm{HR}=9.11, P=.04)$, followed by pattern $4(\mathrm{HR}=6.2, P=$ $.023)$, pattern $3(\mathrm{HR}=4.65, P<.001)$, and pattern 1 (logrank, $P<.001)$. OS with tumor-dominant type NEL did not differ in accordance with CE evolution pattern (logrank, $P=.879$ ) (Fig. 4).

\section{Discussion}

This study analyzed the ability of type of peritumoral NEL and evolution pattern of CE in response to CCRT to predict pseudoprogression, TTP, and OS in patients with IDH wild-type glioblastoma. TTP was found to be shorter and pseudoprogression rate lower in patients with tumor-dominant than edema-dominant type NEL. Of the four evolution patterns of $\mathrm{CE}$, enlarging residual CE after STR (pattern 4) showed shortest TTP while stable residual CE after STR (pattern 2) showed shortest OS. Interestingly, both TTP and OS was better predicted by CE evolution pattern and NEL type than by either alone. TTP and OS could be stratified by CE evolution pattern in patients with edema-dominant, but not tumor-dominant, NEL. Taken together, these results suggested that, when combined with $\mathrm{CE}$ evolution and extent of surgical resection, NEL type improves the prediction of pseudoprogression and helps to further stratify TTP and OS.

Several recent studies have suggested that NEL may be a prognostic marker in patients with glioblastoma [9, 16]. For example, a multicenter study found that high postoperative residual NEL volume was significantly associated with poor overall survival [9]. Histopathologic examination showed that the content of viable tumor cells was higher in non-contrast enhancing tumors than in contrast enhancing tumors or necrotic tumor

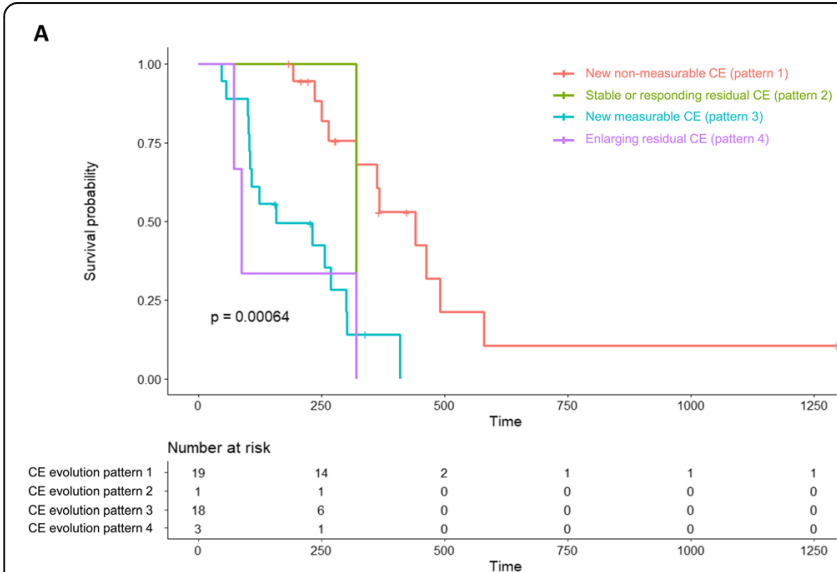

B

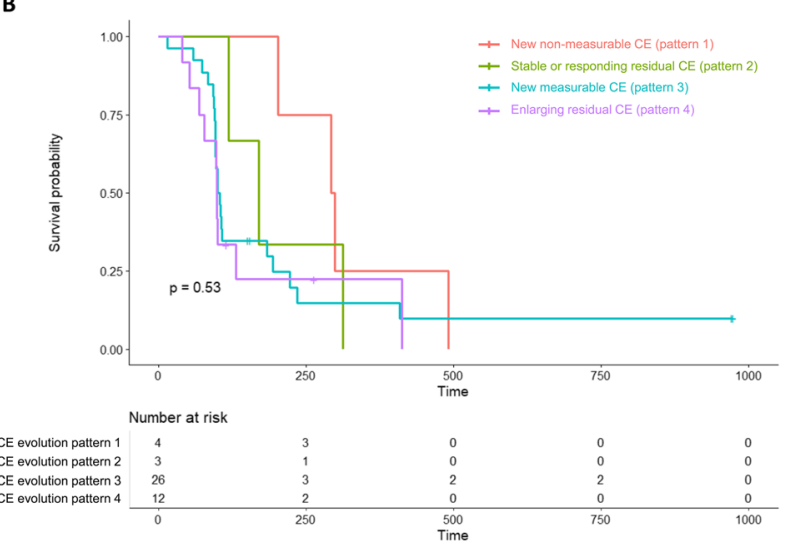

C

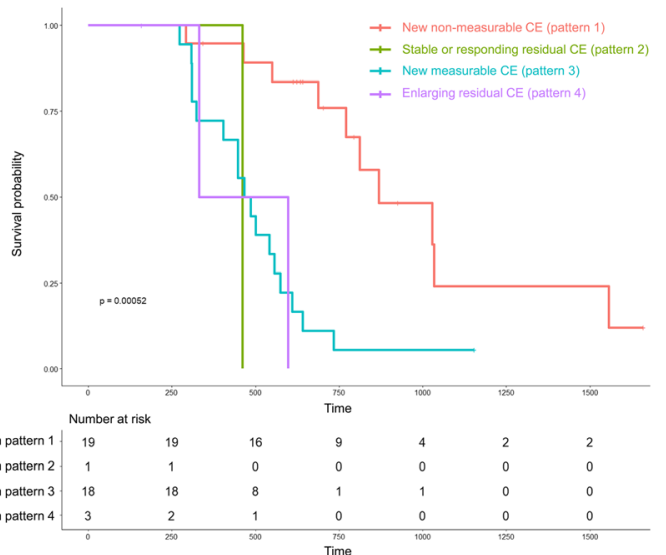

D

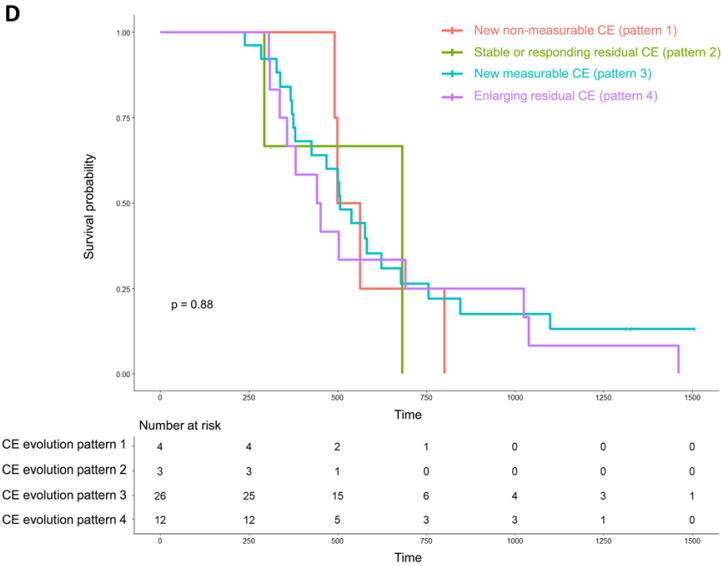

Fig. 4 Prediction of TTP (A, B) and OS (C, D) based on NEL type and CE evolution pattern in patients with IDH wild-type glioblastoma. (A) KaplanMeier analysis of TTP in patients with edema-dominant type NEL stratified by CE evolution pattern (log-rank, $P=.001$ ). (B) Kaplan-Meier analysis of TTP in patients with tumor-dominant type NEL stratified by CE evolution pattern (log-rank, $P=.528$ ). (C) Kaplan-Meier analysis of OS in patients with edema-dominant type NEL stratified by CE evolution pattern (log-rank, $P=.001$ ). (D) Kaplan-Meier analysis of OS in patients with tumor-dominant type NEL stratified by $C E$ evolution pattern (log-rank, $P=$. 879). TTP = time to progression; $O S=$ overall survival; $N E L=$ non-enhancing lesion; $C E=$ contrast enhancement; $\mathrm{IDH}=$ isocitrate dehydrogenase 
components [6]. Nonetheless, association of residual NEL (subdivided into edema- and tumor-dominant types) with treatment remains poorly understood. In our study, patients with tumor-dominant NEL type on preCCRT examination had higher rates of true progression at the appearance of contrast enhancing lesions, as well as shorter TTP. This NEL type was a stronger predictor of pseudoprogression than $\mathrm{CE}$ evolution pattern during CCRT. In the clinical setting, the more aggressive management of NEL, with neurosurgical resection or radiosurgery, has shown greater prognostic benefit than management of the contrast enhancing lesion alone [23, 24]. However, complete resection of NEL is difficult and could increase the risk of postoperative neurological deficits [25]. This study showed the importance of distinguishing tumor-dominant type from edema-dominant type NEL and the selective aggressive management of tumor-dominant type may prolong survival. In addition, morphologic categorization of NEL type showed almost perfect interobserver agreement, suggesting that NEL type can become a useful and reproducible biomarker on anatomical MRI.

We categorized CE evolution pattern in response to CCRT based on surgical extent, change in lesion size, and lesion measurability, all of which are important variables in predicting tumor response and prognosis $[7,18$, 26]. Although extent of residual $C E$ has been associated with survival $[17,18]$, few studies have evaluated both residual $\mathrm{CE}$ and $\mathrm{CE}$ evolution pattern following CCRT. Our study showed that prognosis was worst in patients with residual $\mathrm{CE}$ compared to new $\mathrm{CE}$, in agreement with studies showing the importance of the extent of residual CE $[17,18]$. Combining surgical extent with CE evolution may therefore improve patient prognosis. Our study revealed that the TTP was shortest in patients with enlarging residual CE, while OS was shortest in patients with stable or responding residual CE. We speculated that although enlarging residual $\mathrm{CE}$ could be a direct marker for early tumor progression, pseudoprogression might account for a significant portion of it and affect the long-term outcomes of OS. Tumor heterogeneity of glioblastoma is an important reason for treatment failure, and tumor cells that survive initial therapy mainly cause tumor re-growth or recurrence [27]. The remaining tumor cells in stable or responding CE might lead to treatment resistance and ultimately be responsible for poor patient prognosis.

$\mathrm{CE}$ evolution pattern were significantly associated with prognosis, TTP, and OS in patients with edemadominant, but not tumor-dominant NEL [27]. To our knowledge, no previous studies have investigated the relationship between NEL type and CE evolution pattern, nor assessed the ability of both together to predict prognosis. Our study showed a significant association between NEL type and CE evolution pattern. CE evolution pattern 3 and 4 were observed more frequently in tumor-dominant than edema-dominant type NEL, suggesting that NEL type should be considered when determining CE evolution pattern, and that both should be considered in predicting patient prognosis.

This study had several limitations in addition to those due to its retrospective nature. First, histological confirmation was not possible at the time of radiographic progression because of the invasiveness of these tumors. Second, the number of patients with IDH wild-type glioblastoma was relatively small. Third, this study did not include the results of advanced MRI, including DWI and perfusion-weighted imaging, which increase the diagnostic accuracy of pseudoprogression [28-31]. However, lack of standardization of advanced imaging protocols has prevented their use as imaging biomarkers in multicenter practices. Our study only included the results of conventional MRI, which is generally accepted in multicenter practices. Fourth, volumetric measurement of $\mathrm{CE}$ had not been applied. Further studies are warranted before applying our results to daily clinical practice.

\section{Conclusion}

In conclusion, the addition of peritumoral NEL type to CE evolution pattern improves prediction of pseudoprogression and helps to further stratify TTP and OS in patients with IDH wild-type glioblastoma. Edema-dominant NEL showed a greater association with CE-based survival stratification and pseudoprogression, whereas tumor-dominant NEL was an independent predictor of shorter TTP. This determination of NEL type, together with CE evolution pattern, may further stratify TTP and OS and may become a helpful imaging biomarker for refining RANO criteria.

\section{Abbreviations}

IDH: Isocitrate dehydrogenase; CCRT: Concurrent chemoradiotherapy;

CE: Contrast enhancement; NEL: Non-enhancing lesion; SD: Stable disease;

PD: Progressive disease; TTP: Time to progression; OS: Overall survival

\section{Supplementary Information}

The online version contains supplementary material available at https://doi. org/10.1186/s12885-021-08414-2.

Additional file 1: Supplementary Table 1. Accuracy of a combination of non-enhancing lesions (NEL) type and contrast enhancement (CE) evolution patterns in predicting pseudoprogression. Supplementary Fig. 1. (A) Kaplan-Meier analysis of time to progression (TTP) in patients with tumor-dominant and edema-dominant types of non-enhancing lesions (NEL) inIDH wild-type glioblastoma. The TTP was significantly shorter in patients with tumor-dominant than edema-dominant NEL (hazard ratio $[\mathrm{HR}]=2.03$; log-rank, $P=.005)$.). (B) Kaplan-Meier analysis of overall survival (OS) in patients with tumor-dominant and edema-dominant types of NEL in IDH wild-type glioblastoma. The OS tends to be shorter in patients with tumor-dominant than in patients with edema-dominant NEL, but the difference did not reach statistical significance ( $H R=1.37$, $P=.196)$. Supplementary Fig. 2. (A) Kaplan-Meier analysis of time to progression (TTP) based on contrast enhancement (CE) evolution patterns 
in patients with IDH wild-type glioblastoma. TTP was shortest in patients with enlarging residual CE after STR (subtotal resection) (pattern 4; HR= $4.33, P<.001$ ), followed by new measurable CE after GTR (gross total resection) (pattern $3 ; \mathrm{HR}=3.00, P<.001$ ), stable or responding residual $\mathrm{CE}$ after STR (pattern 2; HR= 2.44, $P=.117$ ), and new non-measurable CE after GTR (pattern 1; $P<.001$ ). (B) Kaplan-Meier analysis of overall survival (OS) based on CE evolution patterns in patients with IDH wild-type glioblastoma. OS was shortest in patients with pattern $2(H R=3.71, P=.009)$, followed by pattern $4(H R=2.74, P=.04)$, pattern $3(H R=2.50, P=.005)$, and pattern $1(P<.001)$.

\section{Acknowledgements}

Not applicable.

\section{Authors' contributions}

All authors reviewed the manuscript. H.H.M.: Manuscript writing. H.S.K.: Manuscript editing, conceptual feedback, and project integrity. J.E.P.: Image analysis and manuscript editing. Y.H.K.: Database construction and data provision. J.H.K.: Clinical/oncologic database curation and oversight. The author(s) read and approved the final manuscript.

\section{Funding}

This research was supported by a National Research Foundation of Korea (NRF) grant funded by the Korean government (MSIP) (grant number: NRF2020R1A2B5B01001707 and NRF-2020R1A2C4001748).

\section{Availability of data and materials}

The datasets generated and/or analysed during the current study are not publicly available due to the hospital's policy but are available from the corresponding author on reasonable request.

\section{Declarations}

\section{Ethics approval and consent to participate}

The protocol for this retrospective study followed the Helsinki declaration and was approved by the institutional review board of Asan Medical Center, Seoul, Korea (IRB No. 2020-0363). Informed consent was waived by the same ethics committee that approved this study (the institutional review board of Asan Medical Center, Seoul, Korea).

\section{Consent for publication}

Not applicable.

\section{Competing interests}

The authors declare that they have no competing interests.

\section{Author details}

'Department of Radiology and Research Institute of Radiology, University of Ulsan College of Medicine, Asan Medical Center, 43 Olympic-ro 88, 86 Asanbyeongwon-Gil, Songpa-Gu, Seoul 05505, Republic of Korea.

${ }^{2}$ Department of Neurosurgery, University of Ulsan College of Medicine, Asan Medical Center, 86 Asanbyeongwon-Gil, Songpa-Gu, Seoul 05505, Republic of Korea.

Received: 8 February 2021 Accepted: 24 May 2021

Published online: 01 June 2021

\section{References}

1. Leao D, Craig P, Godoy L, Leite C, Policeni B. Response assessment in neurooncology criteria for gliomas: practical approach using conventional and advanced techniques. Am J Neuroradiol. 2020;41(1):10-20. https://doi.org/1 0.3174/ajnr.A6358

2. Pope WB, Hessel C. Response assessment in neuro-oncology criteria: implementation challenges in multicenter neuro-oncology trials. AJNR Am J Neuroradiol. 2011:32(5):794-7. https://doi.org/10.3174/ajnr.A2582.

3. Yang D. Standardized MRI assessment of high-grade glioma response: a review of the essential elements and pitfalls of the RANO criteria. Neurooncology practice. 2016;3(1):59-67. https://doi.org/10.1093/nop/npv023.

4. Wen PY, Macdonald DR, Reardon DA, Cloughesy TF, Sorensen AG, Galanis E, et al. Updated response assessment criteria for high-grade gliomas: response assessment in neuro-oncology working group. J Clin Oncol. 2010; 28(11):1963-72. https://doi.org/10.1200/JCO.2009.26.3541.

5. Lasocki A, Gaillard F, Tacey MA, Drummond KJ, Stuckey SL. The incidence and significance of multicentric noncontrast-enhancing lesions distant from a histologically-proven glioblastoma. J Neuro-Oncol. 2016;129(3):471-8. https://doi.org/10.1007/s11060-016-2193-y.

6. Eidel O, Burth S, Neumann J-O, Kieslich PJ, Sahm F, Jungk C, et al. Tumor infiltration in enhancing and non-enhancing parts of glioblastoma: a correlation with histopathology. PLoS One. 2017;12(1):e0169292. https://doi. org/10.1371/journal.pone.0169292.

7. Grabowski MM, Recinos PF, Nowacki AS, Schroeder JL, Angelov L, Barnett $\mathrm{GH}$, et al. Residual tumor volume versus extent of resection: predictors of survival after surgery for glioblastoma. J Neurosurg. 2014;121(5):1115-23. https://doi.org/10.3171/2014.7.JNS132449.

8. Jain R, Poisson LM, Gutman D, Scarpace L, Hwang SN, Holder CA, et al. Outcome prediction in patients with glioblastoma by using imaging, clinical, and genomic biomarkers: focus on the nonenhancing component of the tumor. Radiology. 2014;272(2):484-93. https://doi.org/10.1148/radiol.14131691.

9. Kotrotsou A, Elakkad A, Sun J, Thomas GA, Yang D, Abrol S, et al. Multicenter study finds postoperative residual non-enhancing component of glioblastoma as a new determinant of patient outcome. J Neuro-Oncol. 2018;139(1):125-33. https://doi.org/10.1007/s11060-018-2850-4.

10. Kono K, Inoue Y, Nakayama K, Shakudo M, Morino M, Ohata K, et al. The role of diffusion-weighted imaging in patients with brain tumors. Am J Neuroradiol. 2001;22(6):1081-8.

11. Brandão LA, Shiroishi MS, Law M. Brain tumors: a multimodality approach with diffusion-weighted imaging, diffusion tensor imaging, magnetic resonance spectroscopy, dynamic susceptibility contrast and dynamic contrast-enhanced magnetic resonance imaging. Magnetic Resonance Imaging Clinics. 2013;21(2): 199-239. https://doi.org/10.1016/.j.mric.2013.02.003.

12. Castillo M, Smith JK, Kwock L, Wilber K. Apparent diffusion coefficients in the evaluation of high-grade cerebral gliomas. Am J Neuroradiol. 2001;22(1):60-4.

13. Tien RD, Felsberg GJ, Friedman H, Brown M, MacFall J. MR imaging of highgrade cerebral gliomas: value of diffusion-weighted echoplanar pulse sequences. Am J Roentgenol. 1994;162(3):671-7. https://doi.org/10.2214/ajr.1 62.3.8109520.

14. Lasocki A, Gaillard F. Non-contrast-enhancing tumor: a new frontier in glioblastoma research. Am J Neuroradiol. 2019;40(5):758-65. https://doi. org/10.3174/ajnr.A6025.

15. Pope WB, Sayre J, Perlina A, Villablanca JP, Mischel PS, Cloughesy TF. MR imaging correlates of survival in patients with high-grade gliomas. Am J Neuroradiol. 2005;26(10):2466-74.

16. Lasocki A, Gaillard F, Tacey M, Drummond K, Stuckey S. Incidence and prognostic significance of non-enhancing cortical signal abnormality in glioblastoma. J Med Imaging Radiat Oncol. 2016;60(1):66-73. https://doi. org/10.1111/1754-9485.12421.

17. Ammirati M, Vick N, Liao YL, Ciric I, Mikhael M. Effect of the extent of surgical resection on survival and quality of life in patients with supratentorial glioblastomas and anaplastic astrocytomas. Neurosurgery. 1987;21(2):201-6. https://doi.org/10.1227/00006123-198708000-00012.

18. Lacroix M, Abi-Said D, Fourney DR, Gokaslan ZL, Shi W, DeMonte F, et al. A multivariate analysis of 416 patients with glioblastoma multiforme: prognosis, extent of resection, and survival. J Neurosurg. 2001;95(2):190-8. https://doi.org/10.3171/jns.2001.95.2.0190.

19. Weller M, Van Den Bent M, Tonn JC, Stupp R, Preusser M, Cohen-JonathanMoyal E, et al. European Association for Neuro-Oncology (EANO) guideline on the diagnosis and treatment of adult astrocytic and oligodendroglial gliomas. Lancet Oncol. 2017;18(6):e315-29. https://doi.org/10.1016/S1470-2 045(17)30194-8

20. Brandes AA, Franceschi E, Tosoni A, Blatt V, Pession A, Tallini G, et al. MGMT promoter methylation status can predict the incidence and outcome of pseudoprogression after concomitant radiochemotherapy in newly diagnosed glioblastoma patients. J Clin Oncol. 2008;26(13):21927. https://doi.org/10.1200/JCO.2007.14.8163.

21. Esteller M, Hamilton SR, Burger PC, Baylin SB, Herman JG. Inactivation of the DNA repair gene O6-methylguanine-DNA methyltransferase by promoter hypermethylation is a common event in primary human neoplasia. Cancer Res. 1999:59(4):793-7.

22. Showalter TN, Andrel J, Andrews DW, Curran WJ Jr, Daskalakis C, WernerWasik M. Multifocal glioblastoma multiforme: prognostic factors and patterns of progression. Int J Radiat Oncol Biol Phys. 2007;69(3):820-4. 
23. Li YM, Suki D, Hess K, Sawaya R. The influence of maximum safe resection of glioblastoma on survival in 1229 patients: can we do better than grosstotal resection? J Neurosurg. 2016;124(4):977-88. https://doi.org/10.3171/201 5.5.JNS142087.

24. Duma CM, Kim BS, Chen PV, Plunkett ME, Mackintosh R, Mathews MS, et al. Upfront boost Gamma Knife "leading-edge" radiosurgery to FLAIR MRIdefined tumor migration pathways in 174 patients with glioblastoma multiforme: a 15-year assessment of a novel therapy. J Neurosurg. 2016; 125(Supplement_1):40-9.

25. Pessina F, Navarria P, Cozzi L, Ascolese AM, Simonelli M, Santoro A, et al. Maximize surgical resection beyond contrast-enhancing boundaries in newly diagnosed glioblastoma multiforme: is it useful and safe? A single institution retrospective experience. J Neuro-Oncol. 2017;135(1):129-39. https://doi.org/10.1007/s1 1060-017-2559-9.

26. Albert FK, Forsting M, Sartor K, Adams H-P, Kunze S. Early postoperative magnetic resonance imaging after resection of malignant glioma: objective evaluation of residual tumor and its influence on regrowth and prognosis. Neurosurgery. 1994;34(1):45-61.

27. Osuka S, Van Meir EG. Overcoming therapeutic resistance in glioblastoma: the way forward. J Clin Invest. 2017;127(2):415-26. https://doi.org/10.1172/ JCl89587.

28. Barajas RF Jr, Chang JS, Segal MR, Parsa AT, McDermott MW, Berger MS, et al. Differentiation of recurrent glioblastoma multiforme from radiation necrosis after external beam radiation therapy with dynamic susceptibilityweighted contrast-enhanced perfusion MR imaging. Radiology. 2009;253(2): 486-96. https://doi.org/10.1148/radiol.2532090007.

29. Chu HH, Choi SH, Ryoo I, Kim SC, Yeom JA, Shin H, et al. Differentiation of true progression from pseudoprogression in glioblastoma treated with radiation therapy and concomitant temozolomide: comparison study of standard and high-b-value diffusion-weighted imaging. Radiology. 2013; 269(3):831-40. https://doi.org/10.1148/radiol.13122024.

30. Zhang H, Ma L, Wang $Q$, Zheng X, Wu C, Xu B-n. Role of magnetic resonance spectroscopy for the differentiation of recurrent glioma from radiation necrosis: a systematic review and meta-analysis. Eur J Radiol. 2014; 83(12):2181-9. https://doi.org/10.1016/j.ejrad.2014.09.018.

31. Kim HS, Goh MJ, Kim N, Choi CG, Kim SJ, Kim JH. Which combination of MR imaging modalities is best for predicting recurrent glioblastoma? Study of diagnostic accuracy and reproducibility. Radiology. 2014;273(3):831-43. https://doi.org/10.1148/radiol.14132868.

\section{Publisher's Note}

Springer Nature remains neutral with regard to jurisdictional claims in published maps and institutional affiliations.

Ready to submit your research? Choose BMC and benefit from:

- fast, convenient online submission

- thorough peer review by experienced researchers in your field

- rapid publication on acceptance

- support for research data, including large and complex data types

- gold Open Access which fosters wider collaboration and increased citations

- maximum visibility for your research: over $100 \mathrm{M}$ website views per year

At $\mathrm{BMC}$, research is always in progress.

Learn more biomedcentral.com/submissions 